\title{
Análisis de la mortalidad perinatal en nacidos con peso menor de 1000 gramos en el Hospital San Cecilio de Granada (España) durante el período 1991-2010
}

\author{
Perinatal mortality analysis in newborns with weight under \\ 1000 grams at San Cecilio's Hospital (Granada, Spain) \\ during the 1991-2010 period
}

Dra. Milagros Cruz Martínez ${ }^{a}$, Lic. Rosario Redondo Aguilar ${ }^{a}$, Dra. África Caño Aguilar ${ }^{a}$, Dra. Pilar Carretero Lucena ${ }^{a}$, Dra. Carmen Padilla Vinuesa ${ }^{a}$ y Dra. Ángeles Ruiz Extremera ${ }^{b}$

\section{RESUMEN}

Introducción. La mortalidad perinatal ha disminuido sustancialmente en las últimas décadas. La prematuridad y el bajo peso al nacer son los factores predictivos más fuertemente asociados a esta mortalidad. El objetivo es analizar la evolución de la mortalidad perinatal en los nacidos con peso menor de $1000 \mathrm{~g}$ en los últimos 20 años (1991-2010) y sus causas.

Población y métodos. Estudio observacional-descriptivo de tipo ecológicolongitudinal, sobre 264 nacidos con peso menor de $1000 \mathrm{~g}$ de un total de 56024 nacidos durante el período estudiado. Se calculan las diferentes tasas de mortalidad perinatal específicas por peso. Se aplica el coeficiente de correlación Rho de Spearman para evaluar la relación entre las tasas de mortalidad y los años de estudio, y las pruebas ANOVA y de MannWhitney para comparación de quinquenios y decenios, respectivamente.

Resultados. Se han producido 131 muertes perinatales, 82 de ellas muertes fetales y 49 neonatales precoces. El $64,1 \%$ sucede antes de la semana de gestación 27. Sólo la tasa de mortalidad fetal presenta una disminución estadísticamentesignificativa, aunque la mortalidad perinatal presenta una tendencia al descenso, pero sin alcanzar la significación. Las principales causas inmediatas de óbito son la inmadurez extrema, la hipoxia intrauterina y la infección. Las causas fundamentales relacionadas con la muerte de este grupo de nacidos son la infección por rotura prematura de membranas, la hipertensión materna, la amenaza de parto pretérmino incontrolable y la gemelaridad.

Conclusiones. La disminución de las tasas de mortalidad en este grupo de nacidos está sufriendo un enlentecimiento.

Palabras clave: nacidos con peso extremadamente bajo, mortalidad perinatal.

http:/ /dx.doi.org/10.5546/aap.2013.45

\section{INTRODUCCIÓN Y OBJETIVOS}

La mortalidad perinatal junto con la mortalidad materna son indicadores básicos de salud ${ }^{1}$ que permiten valorar la calidad de los sistemas sanitarios y planificar las mejoras en salud materno-infantil.

Esta mortalidad ha disminuido sustancialmente, aunque en el mundo siguen muriendo más de 7 millones de niños al año durante este período. ${ }^{2}$ Europa muestra cifras que van de 4,5 a $8,7 \%$; ${ }^{3}$ en España, está alrededor del $5 \%$ \%. Esta disminución es debida a la mejora en la salud de la población y a la asistencia sanitaria recibida por las gestantes y los neonatos ${ }^{5} \mathrm{y}$, especialmente, al desarrollo de nuevas tecnologías para el control del bienestar fetal y de los cuidados neonatales.

El bajo peso al nacer (BPN) es el factor individual principal que determina las probabilidades del recién nacido de sobrevivir y tener un crecimiento y desarrollo sanos. Así, la prematuridad y el BPN son los factores predictivos más fuertemente asociados con la mortalidad, existiendo una relación directa entre el peso al nacimiento y la mortalidad perinatal. ${ }^{1,3}$

Esta investigación se centra precisamente en el grupo de nacidos con peso menor de 1000 gramos, catalogado por la Clasificación Estadística Internacional de Enfermedades (CIE)$10,{ }^{6}$ como grupo de extremadamente bajo peso. Mejorar la supervivencia y evitar las causas que favorecen el nacimiento en esta franja de edad y peso influiría en la disminución de la mortalidad perinatal. 
El objetivo del estudio es analizar la evolución de la mortalidad perinatal experimentada en los nacidos con peso menor de $1000 \mathrm{~g}$ en el Hospital Universitario San Cecilio (HUSC) de Granada (España), en el período 1991-2010. Se completa con el análisis de las causas de mortalidad perinatal en este grupo de nacidos y las enfermedades maternas, patología obstétrica y neonatal que contribuyen a esta mortalidad.

\section{POBLACIÓN Y MÉTODOS}

Diseño: Estudio observacional-descriptivo de tipo ecológico longitudinal.

\section{Población}

Desde el 1 de enero de 1991 hasta el 31 de diciembre de 2010 (serie temporal: 20 años), en el HUSC de Granada (España) 264 nacidos tuvieron un peso menor de $1000 \mathrm{~g}$, de un total de 56024 nacimientos. Hubo 131 óbitos perinatales en el grupo de estudio, de un total de 417 muertes perinatales.

Criterios de exclusión: nacidos con peso menor de $500 \mathrm{~g}$ o mayor de $999 \mathrm{~g}$ en el HUSC durante el período 1991-2010.

\section{Método}

Análisis descriptivo de las diferentes variables.

Se calculan las diferentes tasas de mortalidad recomendadas por la $\mathrm{OMS}^{6}$ (fetal, neonatal precoz y perinatal), aplicadas en este estudio a los nacimientos con peso menor de $1000 \mathrm{~g}$.

Se utiliza el coeficiente de correlación Rho de Spearman, para evaluar la relación de las diferentes tasas de mortalidad con el discurrir de los años de estudio (tendencia temporal).

Tras agrupar el período en 4 quinquenios y en 2 decenios se han comparado las medias de las diferentes tasas estudiadas. Se ha utilizado la prueba de ANOVA de un factor ajustado post hoc con el método de Bonferroni, para comparar quinquenios y la prueba de Mann-Whitney para comparar decenios.

Se utiliza el programa estadístico SPSS 15.00, y las diferencias se consideran estadísticamente significativas si $\mathrm{p}<0,05$.

Las autopsias en el hospital son realizadas siempre por la misma persona y el análisis de las causas de mortalidad son analizadas por el mismo equipo; se discute y analiza cada caso y la concordancia entre el diagnóstico clínico y el anatomopatológico.

\section{RESULTADOS}

Se estudian 264 nacidos con un peso comprendido entre 500-999 g (0,47\% del total de nacidos).

Se produjeron 131 óbitos perinatales en el grupo estudiado (500-999 g), lo que supone el 31,41\% del total de muertes perinatales.

\section{Análisis de la mortalidad durante el período perinatal según la semana de gestación (SG) en el momento del nacimiento}

La Tabla 1 refleja la mortalidad, donde se aprecia que antes de la SG 24 es cuando más muertes perinatales se producen $(38,9 \%)$, seguida por las producidas entre la SG $24-26(25,2 \%)$.

$\mathrm{Al}$ valorar el momento de la muerte perinatal, se observa que las muertes fetales son igualmente más frecuentes antes de la SG 24 (40,2\%). Este hecho también se observa en las muertes neonatales precoces (36,7\% antes de la SG 24$)$, debido fundamentalmente a que todos los nacidos con inmadurez extrema fallecen en los primeros días.

\section{Análisis de las tasas de mortalidad (Figura 1)}

Las 131 muertes perinatales se distribuyen en 82 óbitos fetales y 49 neonatales precoces.

La tasa de mortalidad fetal presenta una media de 308,66 \pm 133,85\% , iniciándose en el año 1991

TABla 1. Distribución de la mortalidad en el período perinatal en nacidos con peso menor $1000 \mathrm{~g}$, según semana de gestación en el momento del nacimiento

\begin{tabular}{lccc}
\hline Semana gestación & Muertes perinatales n (\%) & Muertes fetales n (\%) & Muertes neonatales precoces n (\%) \\
\hline$<24$ & $51(38,9)$ & $33(40,2)$ & $18(36,7)$ \\
$24-26$ & $33(25,2)$ & $18(22)$ & $15(30,6)$ \\
$27-28$ & $29(22,1)$ & $19(23,2)$ & $10(20,4)$ \\
$29-30$ & $9(6,9)$ & $7(8,5)$ & $2(4,1)$ \\
$31-32$ & $5(3,8)$ & $4(4,9)$ & $1(2,1)$ \\
$>32$ & $1(0,8)$ & $1(1,2)$ & $0(0)$ \\
Desconocido & $3(2,3)$ & $0(0)$ & $3(6,1)$ \\
Total & $131(100)$ & $82(62,6)$ & $49(37,4)$ \\
\hline
\end{tabular}

Solo se consideran los nacidos con peso entre 500-999 g, según recomienda la OMS para el grupo de extremadamente bajo peso. 
con $500 \%$ y terminando en el año 2010 con $250 \%$. A pesar de la amplia latitud $(0 \%-555,5 \%$ o), se ha conseguido alcanzar un descenso significativo (Rho: -0,538) ( $\mathrm{p}=0,015)$.

La tasa de mortalidad neonatal precoz presenta una media de $300,84 \pm 206,17 \%$, igualmente con amplios intervalos según los años estudiados (0$800 \%$ ), hecho que ha contribuido a que no se alcance la significación (Rho: 0,065$)(p=0,784)$.

La tasa de mortalidad perinatal es de $508,12 \pm 191,76 \%$, también con un amplio intervalo $(0-857,14 \%)$, aunque presenta una tendencia al descenso (Rho: $-0,244)$, ésta no es significativa $(p=0,3)$.
Al distribuir el período en 4 quinquenios y comparar sus medias (Tabla 2), se observa que sólo la mortalidad fetal experimenta diferencias estadísticas significativas entre los quinquenios $1^{\circ}$ (1991-1995) y $3^{\circ}$ (2001-2005). No existen diferencias significativas ni para la mortalidad neonatal precoz, ni para la mortalidad perinatal.

Para completar el estudio se analizó si existen diferencias entre las medias de las diferentes tasas en los dos decenios que componen el estudio ( $\mathrm{Ta}-$ bla 3), y se halló que sólo la mortalidad fetal experimenta diferencias estadísticamente significativas

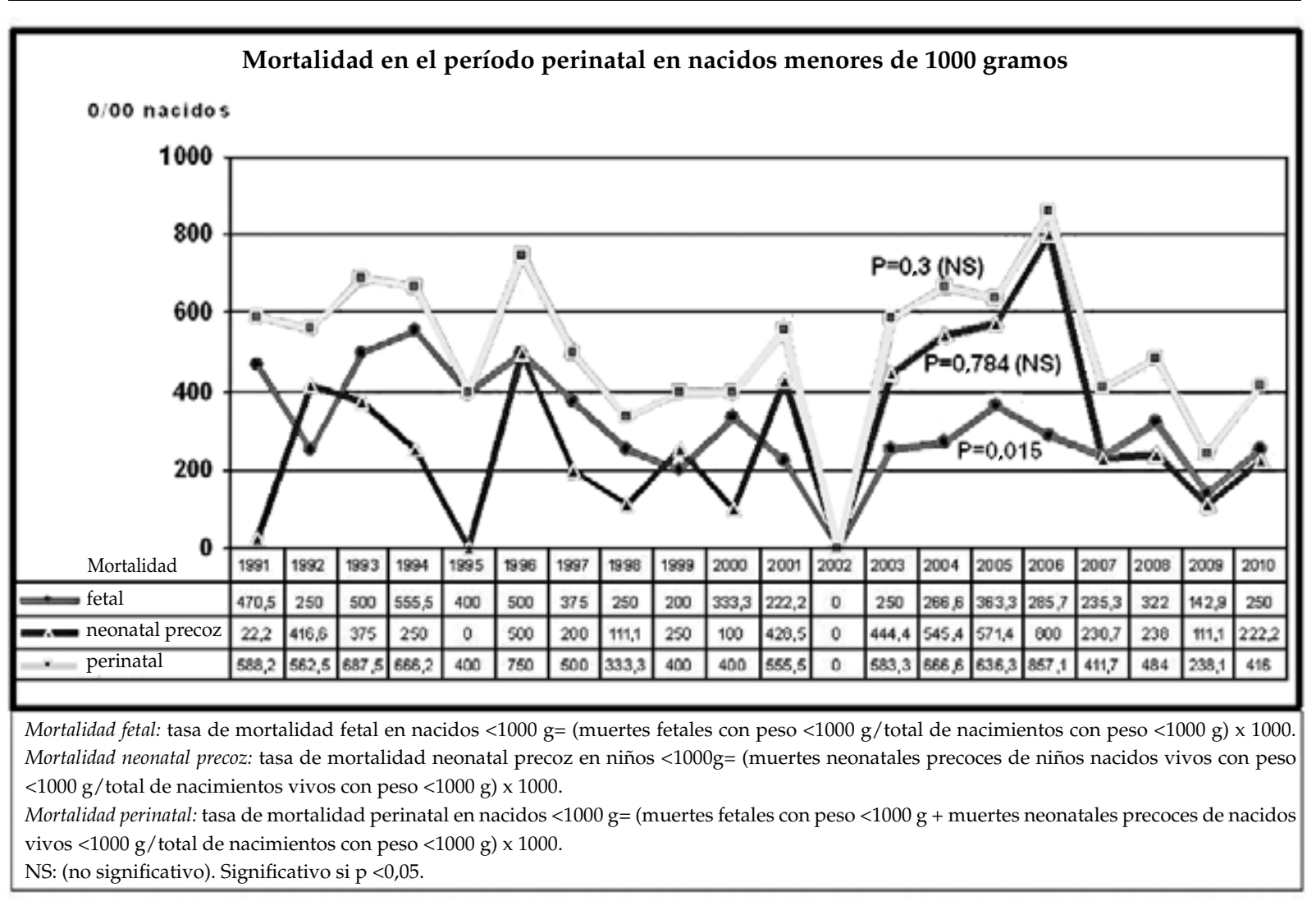

Tabla 2. Análisis de las tasas de mortalidad en el período perinatal distribuidas en quinquenios. Prueba de ANOVA

\begin{tabular}{lccccc}
\hline & $\begin{array}{c}\mathbf{1 9 9 1 - 1 9 9 5} \\
\text { media } \pm \text { DE }\end{array}$ & $\begin{array}{c}\mathbf{1 9 9 6 - 2 0 0 0} \\
\text { media } \pm \text { DE }\end{array}$ & $\begin{array}{c}\mathbf{2 0 0 1 - 2 0 0 5} \\
\text { media } \pm \text { DE }\end{array}$ & $\begin{array}{c}\mathbf{2 0 0 6 - 2 0 1 0} \\
\text { media } \pm \text { DE }\end{array}$ & p \\
\hline Tasa mortalidad fetal & $435,21 \pm 117,75^{*}$ & $331,66 \pm 116,4$ & $220,50 \pm 134,26^{*}$ & $247,27 \pm 67,43$ & $0,033^{*}$ \\
Tasa mortalidad neonatal precoz & $252,77 \pm 163,27$ & $232,22 \pm 162,18$ & $397,97 \pm 230,92$ & $320,41 \pm 273,07$ & 0,612 \\
Tasa mortalidad perinatal & $580,97 \pm 113,83$ & $476,66 \pm 163,97$ & $488,38 \pm 276,46$ & $486,48 \pm 222,38$ & 0,834 \\
\hline
\end{tabular}

Tras la aplicación de la prueba de ANOVA de un factor con la corrección post hoc de Bonferroni, solo se encuentran diferencias estadísticamente significativas entre los quinquenios 1991-1995 y 2001-2005 para la mortalidad fetal.

DE: desviación estándar; $\mathrm{p}$ : valor significación; *:p<0,05. 


\section{Causas de muerte}

Se han realizado 88 autopsias perinatales; de ellas, 59 eran óbitos fetales y 29 eran decesos neonatales precoces. Se halló concordancia diagnóstica entre la clínica y los resultados anatomopatológicos en un $86 \%$ en el grupo de mortalidad fetal y en un $80 \%$ en el grupo de mortalidad neonatal; en el resto, la necropsia permitió establecer el diagnóstico de la causa de muerte.

Las causas inmediatas de muerte (Tabla 4) son, principalmente, la inmadurez extrema $(23,7 \%)$, la hipoxia intrauterina $(22,9 \%)$ y la infección $(15,3 \%)$. La enfermedad de membrana hialina se ha presentado en 9 casos $(6,9 \%)$ y las malformaciones en $11(8,4 \%)$. Otras patologías han sido 4 casos de transfusión feto-fetal y 7 con crecimiento intraútero retardado. No ha habido ninguna hemorragia intraventricular como causa inmediata de óbito.

La hipoxia intrauterina y la asfixia intraparto ha disminuido a lo largo de todo el período estudiado (25,7 a $20 \%)$; sin embargo, el número de muertes relacionadas con la infección $(9,1 \%$ a $21,5 \%)$ y los trastornos por gestación corta y bajo peso: inmadu- rez extrema $(19,7 \%$ a $27,7 \%)$ han aumentado en la última década. La isoinmunización y la transfusión feto-fetal han desaparecido como causa de muerte en este grupo en el último decenio.

Al considerar las causas fundamentales de muerte perinatal (Tabla 5), la rotura prematura de membranas (RPM) que ha favorecido la corioamnionitis/sepsis apareció en 31 casos $(23,7 \%)$.

La patología relacionada con el parto, fundamentalmente la dinámica uterina no controlable que ocasiona parto pretérmino, fue el origen de 25 decesos $(19,1 \%)$ y se convirtió en la $3^{\mathrm{a}}$ causa fundamental de muerte, tras la infección y la afectación del feto por condiciones de la placenta, el cordón umbilical y las membranas.

Entre las patologías maternas asociadas, la principal fue la hipertensión (22 casos), que resultó la causa fundamental que produjo la muerte en 19 de ellos. La patología placentaria surgió en 20 casos: 14 fueron desprendimientos de placenta, asociada en ocasiones $(10,7 \%)$ a hipertensión materna; los otros casos han sido metrorragia por placenta previa o infartos

Tabla 3. Análisis de las tasas de mortalidad en el período perinatal distribuidas en decenios. Prueba de Mann-Whitney

\begin{tabular}{lcccc}
\hline & $\begin{array}{c}\mathbf{1 9 9 1 - 2 0 0 0} \\
\text { media } \pm \text { DE }\end{array}$ & $\begin{array}{c}\mathbf{2 0 0 1 - 2 0 1 0} \\
\text { media } \pm \text { DE }\end{array}$ & U & p \\
\hline Tasa Mortalidad fetal* & $384,44 \pm 123,14$ & $233,88 \pm 101,15$ & 19,00 & $0,019^{*}$ \\
Tasa Mortalidad neonatal precoz & $242,49 \pm 153,80$ & $359,19 \pm 241,89$ & 35,00 & 0,256 \\
Tasa Mortalidad perinatal & $528,81 \pm 143,98$ & $487,43 \pm 236,53$ & 47,50 & 0,850 \\
\hline
\end{tabular}

U: valor U de la prueba de Mann-Whitney; p: valor significación; *:p<0,05.

TABLA 4. Causa inmediata de muerte perinatal en nacidos menores $1000 \mathrm{~g}$. CIE-9

\begin{tabular}{lccc}
\hline & 1991-2000 n (\%) & 2001-2010 n (\%) & Total n (\%) \\
\hline Crecimiento intrauterino restringido y desnutrición del feto (764) & $2(3,1)$ & $5(7,7)$ & $7(5,3)$ \\
Trastorno por gestación corta y bajo peso (765) & $13(19,7)$ & $18(27,7)$ & $31(23,7)$ \\
Traumatismos del nacimiento (767) & $2(3,1)$ & 0 & $2(1,5)$ \\
Hipoxia intrauterina y asfixia intraparto (768) & $17(25,7)$ & $13(20)$ & $30(22,9)$ \\
Síndrome de distrés respiratorio neonatal (769) & $3(4,5)$ & $6(9,2)$ & $9(6,9)$ \\
Infecciones específicas del período perinatal (771) & $6(9,1)$ & $14(21,5)$ & $20(15,3)$ \\
Hemorragia feto y neonato (772) & $7(10,6)$ & 0 & $7(5,3)$ \\
Enfermedad hemolítica del neonato por isoinmunización (773) & $3(4,5)$ & 0 & $3(2,3)$ \\
Feto y neonato afectado por embarazo múltiple & & $4(6,1)$ & 0 \\
(síndrome de transfusión feto-fetal) (761.5) & $6(9,1)$ & $5(7,7)$ & $4(3,1)$ \\
Malformaciones congénitas, deformidades y anomalías cromosómicas $(740-759)$ & $3(4,5)$ & $4(6,2)$ & $7(5,3)$ \\
Desconocida & $66(50,4)$ & $65(49,6)$ & $131(100)$ \\
Total & &
\end{tabular}

La causa inmediata es aquella que ha producido la muerte en el feto o recién nacido.

$\%=$ porcentaje sobre el total de muertes perinatales. 
placentarios. La patología del cordón umbilical (circulares, prolapsos de cordón) fue la causa fundamental en 9 casos.

Es importante señalar que 31 casos eran óbitos procedentes de gestaciones múltiples (21 gemelares y 10 en gestaciones de más de dos fetos), 7 casos entre 1991-2000 y 24 entre 2001-2010. En estas gestaciones gemelares, las causas fundamentales más frecuentes de muerte han sido la dinámica uterina no controlable que ocasionó parto pretérmino (12 casos) y la rotura prematura de membranas (RPM) que causó infección (8 casos).

\section{DISCUSIÓN}

Los estudios sobre mortalidad perinatal presentan múltiples dificultades debido al problema de establecer definiciones y criterios diagnósti$\cos ^{7}$ al problema del infrarregistro ${ }^{8-13}$ que impide realizar estadísticas "exactas" y al problema del análisis de las causas, pues no existe un sistema de clasificación universal aplicable a las muertes perinatales, ya que en ellas se implican causas dependientes de la madre, del feto o el recién nacido (o ambos) y del propio embarazo o del parto (o ambos), que a veces son difíciles de diferenciar y clasificar., 74,15

El estudio de la mortalidad en el grupo de estudio es aún más arduo, pues el problema del infrarregistro es de mayor magnitud. ${ }^{8-13}$ Incluso en países desarrollados, no se han establecido sistemas de registro y análisis comunes de la mortalidad perinatal; ni siquiera dentro de cada país existen sistemas de registro equiparables, que servirían para el estudio de esta mortalidad y para poder establecer comparaciones reales entre hospitales, comunidades y países.
En España, algunas investigaciones ${ }^{8-10}$ estiman $^{-1}$ en un $24-35 \%$ la magnitud de la infradeclaración de la mortalidad perinatal nacional; y relacionan algunas circunstancias con este fenómeno, como son el bajo peso al nacer, la edad gestacional temprana y criterios legales o definiciones poco claras en la declaración de estas muertes. Es precisamente el grupo de nacidos que se analiza en este trabajo el que cumple todas estas circunstancias.

A pesar de estas dificultades, las organizaciones sanitarias reconocen que los nacidos con BPN y nacidos pretérmino, especialmente los menores de $1000 \mathrm{~g}$, son los de mayor morbimortalidad. ${ }^{1,3}$ Este grupo, aunque supone un porcentaje muy bajo de los nacimientos totales $(0,7 \%){ }_{,}^{16}$ contribuye con el $20-50 \%$ de la mortalidad durante el primer año de vida. ${ }^{16-18}$

En Europa, ${ }^{3}$ este grupo de nacidos ocasiona el $29,3 \%$ de los óbitos perinatales en esta investigación es algo más elevado (31,41\%), quizás, por el sesgo del infrarregistro y el sesgo que presentan las estadísticas europeas al ser estimaciones inferidas de datos proporcionados por algunos hospitales de cada país.

Según los datos oficiales del Instituto Nacional de Estadística (INE) de España, ${ }^{4}$ la tasa de mortalidad fetal de este grupo presenta un intervalo de $207,9 \%$ - $356,6 \%$; quizás, el problema del infrarregistro $^{8-11}$ en el orden nacional puede contribuir a que la mortalidad fetal de esta investigación presente cifras más altas.

Al analizar los resultados, la fluctuación de las tasas en algunos años, con el amplio intervalo que presentan, ha influido sin duda en la falta de significación estadística de algunas de ellas. A pesar de que la mortalidad fetal sí presenta una

TABla 5. Causa fundamental de muerte perinatal en nacidos menores $1000 \mathrm{~g}$. CIE-9

\begin{tabular}{|c|c|c|c|}
\hline & $1991-2000 \mathrm{n}(\%)$ & $2001-2010$ n $(\%)$ & Total n (\%) \\
\hline Feto y neonato afectado por enfermedad materna $(760)^{*}$ & $11(16,7)$ & $8(12,3)$ & $19(14,5)$ \\
\hline Feto y neonato afectado por rotura prematura de membranas (761) & $12(18,2)$ & $19(29,2)$ & $31(23,7)$ \\
\hline $\begin{array}{l}\text { Feto y recién nacido afectados por complicaciones de la placenta, } \\
\text { cordón umbilical y de las membranas ( } 762)\end{array}$ & $13(19,7)$ & $16(24,6)$ & $29(22,1)$ \\
\hline $\begin{array}{l}\text { Feto y recién nacidos afectados por otras complicaciones } \\
\text { del trabajo de parto y del parto ( } 763 \text { ) }\end{array}$ & $12(18,2)$ & $13(20)$ & $25(19,1)$ \\
\hline Enfermedad originada en el período perinatal (764-779) & $11(16,7)$ & $7(10,8)$ & $18(13,8)$ \\
\hline Desconocida & $7(10,5)$ & $2(3,1)$ & $9(6,8)$ \\
\hline Total & $66(50,4)$ & $65(49,6)$ & $131(100)$ \\
\hline
\end{tabular}

La causa fundamental es aquella afección/enfermedad del feto o recién nacido, o afección/enfermedad principal de la madre que afecta al recién nacido o feto, o complicaciones de los anexos ovulares que ha contribuido a la muerte perinatal.

$\%=$ porcentaje sobre el total de muertes perinatales.

* = todos los casos han sido por hipertensión materna. 
disminución estadísticamente significativa, no así la mortalidad neonatal precoz ni la mortalidad perinatal, aunque ésta presenta un ligero descenso, aunque sin significación. Este enlentecimiento del descenso de las tasas de mortalidad podría, quizás, deberse a estar llegando a cifras difíciles de disminuir. Este hecho ya ha sido apuntado en algunas investigaciones ${ }^{19}$ que sugieren la existencia de unos "límites biológicos" por debajo de los cuales sería extremadamente difícil conseguir reducciones, con los conocimientos científicos actuales. ${ }^{20}$

Además, es inevitable que, en ocasiones, se produzcan situaciones que conducen a la muerte fetal, como son los accidentes del cordón umbilical en las gestaciones pretérmino.

Por otra parte, hay patologías maternas ${ }^{21,22} \mathrm{O}$ complicaciones propias del embarazo (hipertensión, problemas placentarios, etc.), que son difíciles de disminuir aunque sí se pueden controlar más exhaustivamente, ${ }^{23}$ y que hacen que aumenten los partos yatrógenos de recién nacidos pretérmino.

De hecho, en este estudio, la hipertensión materna es la patología materna que más contribuye a esta mortalidad. Algunos estudios ${ }^{24}$ refieren que la hipertensión asociada con parto pretérmino es la principal causa de muerte neonatal precoz y de mortalidad infantil, no solo por la necesidad de tener que provocar un parto pretérmino, sino porque la hipertensión puede producir disfunción placentaria que ocasiona complicaciones fetales y placentarias ${ }^{25}$ que llevan a la muerte fetal o neonatal. Hasta ahora, todas las intervenciones para conseguir prevenir la hipertensión en el embarazo han sido poco eficaces.

En el análisis de las causas de muerte, surge el problema sobre la definición y clasificación de las causas, pues las diferentes investigaciones al respecto no las analizan del mismo modo, ${ }^{12,13}$ superponiéndose causas inmediatas con fundamentales, hecho ya comentado en diferentes estudios, ${ }^{7,14,26}$ por lo cual es necesario establecer criterios homogéneos y contrastados que sirvan en el futuro como guía de actuación y registro. ${ }^{15}$

Los trastornos por gestación corta y bajo peso (inmadurez extrema) suponen en esta investigación la causa inmediata de muerte más frecuente $(23,7 \%)$, que además ha ido creciendo con el avance de los años (1991-2000: 19,7\% y 2001-2010: $27,7 \%$ ), hecho que ha coincidido con un aumento de las gestaciones múltiples en esta última década en nuestro centro. Esta circunstancia quizás haya ocasionado el elevado porcentaje de esta causa de muerte en este estudio (23,7\%), frente a lo referido por algunos autores; ${ }^{17}$ aunque la mayoría de las investigaciones muestran porcentajes similares ${ }^{15,27}$ a los resultados presentados, convirtiéndose la inmadurez extrema en una de las causas inmediatas principales de muerte. ${ }^{26}$

Las malformaciones graves no constituyeron una causa importante de mortalidad (solo 11 casos) en este grupo de nacidos. Este hecho es debido a los avances tecnológicos y a la formación específica de los profesionales, que permiten su diagnóstico prenatal antes de la semana de gestación 22, lo cual posibilita la toma de decisiones acerca de la interrupción del embarazo. El bajo porcentaje de malformaciones $(8,4 \%)$ en este estudio contrasta con lo referido por otros autores $(14 \%) .{ }^{17,28}$

En algunas investigaciones, ${ }^{17,29}$ la hemorragia intraventricular es una de las primeras causas de muerte en este grupo de nacidos; en el presente estudio no hay ningún caso, debido quizás al uso protocolizado de corticoides para la maduración pulmonar fetal, que también proteje al sistema nervioso central. La enfermedad de la membrana hialina, que es otra causa inmediata importante de mortalidad, en esta investigación supone el $6,9 \%$ de las muertes, que contrasta con lo comunicado por otros autores. ${ }^{17}$

La RPM pretérmino sigue siendo uno de los grandes problemas de la obstetricia actual. ${ }^{30,31} \mathrm{En}$ este estudio ha contribuido a la muerte de estos nacidos en 31 casos (23,7\%), acompañada de sospecha de corioamnionitis en la gran mayoría de ellos, hechos que condicionaron que en algunas de estas gestaciones se decidiera la terminación del embarazo (parto pretérmino yatrógeno), a pesar, a veces, de la gran inmadurez. La infección/ sepsis/choque séptico supone una de las principales causas de muerte, ${ }^{32}$ con amplias variaciones $(14,3-21,4 \%),{ }^{17,28}$ en algunas investigaciones llega al $53,7 \%{ }^{29}$ En esta investigación aparece en el $15,3 \%$ y es la tercera causa de muerte, tras los trastornos por gestación corta y bajo peso (inmadurez extrema) y la hipoxia intrauterina y asfixia intraparto.

Por lo tanto, la prevención de la RPM pretérmino $^{33}$ y sus consecuencias deben ser objeto de investigación en obstetricia. En el HUSC se administra de modo protocolizado progesterona en gestantes con riesgo de parto pretérmino, corticoides ante la amenaza de un parto pretérmino y antibióticos en casos de RPM, esta última indicación puede haber contribuido a la menor incidencia de infección como causa de muerte respecto de la referida en otros estudios. 
El problema de la gemelaridad va en aumento por el avance de las técnicas de reproducción asistida. ${ }^{34}$ Es un hecho que la morbimortalidad es de 3 a 7 veces más alta que en gestaciones simples; ${ }^{35}$ estudios recientes, ${ }^{29}$ indican que la mortalidad perinatal es 3,6 veces mayor y la mortalidad fetal 5,9 veces. Estas altas tasas de morbimortalidad son debidas a la elevada incidencia de complicaciones anteparto, parto pretérmino e insuficiencia uteroplacentaria; ${ }^{35}$ existe mayor morbimortalidad materna y aumento de la mortalidad infantil, con incremento del gasto sanitario y del costo afectivo familiar que conlleva.

En el presente estudio los mortinatos producto de gestaciones múltiples suponen el 23,7\% de la mortalidad y el número de casos se ha triplicado en la última década (7 a 24).

\section{CONCLUSIONES}

El grupo de nacidos menores de $1000 \mathrm{~g}$ supone el $0,47 \%$ del total de nacidos; sin embargo, ocasionan más del $30 \%$ de la mortalidad perinatal.

Las principales causas inmediatas de muerte son la inmadurez extrema, la hipoxia intrauterina y la infección.

Las gestaciones múltiples, la hipertensión materna, la infección por rotura prematura de membranas y la dinámica uterina que ocasiona parto pretérmino, son las causas fundamentales relacionadas con la muerte de este grupo de nacidos.

La disminución de las tasas de mortalidad en este grupo de nacidos está sufriendo un enlentecimiento.

\section{BIBLIOGRAFÍA}

1. Organización Mundial de la Salud. Estadísticas Sanitarias Mundiales. 2011. [Acceso: 27 de julio 2012]. Disponible en: http://www.who.int/whosis/whostat/ES_whs2011_full. pdf.

2. Zupan J. Perinatal mortality in developing countries. $N$ Eng J Med 2005;352(20):2047-8.

3. EURO-PERISTAT Project, with SCPE, EUROCAT, EURONEOSTAT. European Perinatal Health Report. 2008. [Acceso: 27jul 2012]. Disponible en: http//www.sante.public. $\mathrm{lu} /$ publications/sante-file-vie/petite-enfance/europeanperinatal-health-report/european-perinatal-health-report. pdf.

4. Anuario Estadístico de España. Años 2000-2009. Instituto Nacional deEstadística. Madrid. [Acceso: 6 de agosto 2012]. Disponible en: http://www.ine.es/proyser/pubweb/ anuarios_mnu.htm.

5. Carroli G, Villar J, Piaggio G, Khan-Neelofur D, et al. WHO systematic review of randomised controlled trials of routine antenatal care. Lancet 2001;357(9268):1565-70.

6. OMS. Presentación estadística. En: Clasificación Estadística Internacional de Enfermedades y Problemas Relacionados con la Salud. 10a Revisión Clínica. Vol 2: Manual de Instrucciones. Washington: Organización Panamericana de la Salud; 1997. Págs.125-39.
7. OMS. Certificación Médica de Causa de Defunción. 4ta. ed. Ginebra: Organización Mundial de la Salud, 1980.

8. Mosquera C, González-Rico M. Calidad del registro de muertes perinatales. Asturias, 1986-1990. Gac Sanit. 1994;8(42):112-6.

9. FerrandoJ, BorellC, Ricart M, Plasència A. Infradeclaración de la mortalidad perinatal: experiencia de 10 años de vigilancia activa en Barcelona. Med Clin (Barc) 1997;108(9):3305 .

10. Revert M, Rué M, Moreno C, Pérez G, et al. Análisis del infrarregistro de mortalidad perinatal y sus factores asociados en una región sanitaria de Cataluña. Gac Sanit 1998;12:6370.

11. Freitas A, Puigdefàbregas A, Gispert R, Barés M, et al. La mortalidad perinatal según 2 fuentes de información. Gac Sanit 2008;22(3):278-81.

12. Castilla J, Moreno C, Eguino E. Validez de las Estadísticas de Mortalidad infantil en Navarra. Gac Sanit 1992;6:153-6.

13. Cirera l, Martínez C, Salmerón D, Navarro C. Subcertificación de las muertes perinatales en obstetricia y neonatología. An Pediatr 2008;69(2):129-33.

14. Tosca R, Aguilera R, Bellido J y Grupo de Estudios Neonatales de la Comunidad Valenciana (GEN-CV). Causas de mortalidad neonatal en la Comunidad Valenciana. An Esp Pediatr 2002;57(6):565-9.

15. Santos JG, Gallego MJ, Imaz A, Martínez JV, et al. Mortalidad neonatal del Hospital Universitario de Valladolid (HUV) en la década de los 90. Bol Pediatr 2004;44:113-9.

16. Suárez A, Rodríguez D. Morbimortalidad en neonatos de muy bajo peso en el Hospital Yvan Portuondo. Estudio de 5 años (2003-2007). [Acceso: 1 de septiembre 2011]. Disponible en: http://www.monografias.com/trabajos-pdf4/ morbimortalidad-neonatos-muy-peso/morbimortalidadneonatos-muy-peso.pdf.

17. Molina OR, Regalado A. Recién nacido de peso extremo. Rev Cubana Obstet Ginecol 2010;36(2)165-75.

18. Bosch S, López A, Melchor I, Ibáñez I, et al. Informe Registro de Mortalidad Perinatal de la Comunidad Valenciana, 2006-2007. Conselleria de Sanitat. Generalitat Valenciana, 2009.

19. Ruiz M, Nieto AM. Evolución de la mortalidad infantil, neonatal y postneonatal en Andalucía, 1975-1998. Rev Esp Salud Pública 2003;77:363-71.

20. Rada D, Cotero A, Centeno C, Valls A. Evolución de la mortalidad neonatal y perinatal en los hospitales del Grupo de Estudios Neonatales Vasco-Navarro (GEN-VN) en el período 2000-2006. An Pediatr (Barc) 2009;70(2):143-50.

21. Clowse M, Magder LS, Witter F, Petri M. Early Risk Factors for Pregnancy Loss in Lupus. Obstet Gynecol 2006;107:2939.

22. Empson M, Lassere M, Craig J, Scott J. Prevention of recurrent miscarriage for women with antiphospholipid antibody or lupus anticoagulant. Cochrane Database Syst Rev 2005:CD002859.

23. Menezes EV, Yakoob MY, Soomro T, Haws RA, et al. Reducing stillbirths: prevention and management of medical disorders and infections during pregnancy. BMC Pregnancy and Childbirth 2009;9(Suppl 1):S4.

24. Villar J, Ezcurra EJ, Gurtner de la Fuente V, Campodonico L. Preterm delivery syndrome: the unmet need. Res Clin Forums 1994;16:9-39.

25. Sibai BM. Caring for women with hypertension in pregnancy. JAMA 2007;298(13):1566-8.

26. Cruz E, Lapresta M, Crespo R, José Y, et al. Análisis de la mortalidad perinatal, Hospital Miguel Servet, Zaragoza, España: 2000-2009. Rev Chil Obstet Ginecol 2011;76(1):3-9.

27. Rivera MA, Hernández M, Hernández G, Llano I, et al. Análisis de la mortalidad neonatal precoz en el Institu- 
to Nacional de Perinatología (1991-2001). Perinatol Reprod Hum 2005;19:13-21.

28. Yllescas-Medrano E, García-Graullera MG, Fernández-Carrocera LA. Valoración del riesgo de morbi-mortalidad en recién nacidos con peso $\leq \mathrm{de} 1,500 \mathrm{~g}$, en una unidad hospitalaria de tercer nivel. Perinatol Reprod Hum 2005;19:87-93.

29. Centre for Maternal and Child Enquiries (CMACE) Perinatal Mortality 2008: United Kingdom. CMACE: London; 2010.

30. Saavedra D, Valdés S, Bardales J, Essien J, et al. Morbimortalidad perinatal de la rotura prematura de membrana en el embarazo pretérmino. Clin Invest Gin Obst 2006;33(3):102-6.

31. Ananth CV, Oyelese Y, Srinivas N, Yeo L, et al. Preterm Premature Rupture of Membranes, Intrauterine Infection, and oligohydramnios: Risk Factors for Placental Abruption. Obstet Gynecol 2004;104:71-7.
32. Moraes M, Cancela MJ, Repetto M, Gutiérrez C, et al. Corioamnionitis histológica en el recién nacido menor de 1.000 gramos. Incidencia y resultados perinatales Rev Soc Bol Ped 2008;47(1):53-9.

33. Barros FC, Bhutta ZA, Batra M, Hansen TN, et al. Global report on preterm birth and stillbirth (3 of 7): evidence for efectiveness of interventions. BMC Pregnancy Childbirth 2010;10(Suppl 1):S3.

34. Contribution of assisted reproductive technology and ovulation-inducing drugs to triplet and higher-order multiple births-United States, 1980-1997. MMWR Morb Mortal Wkly Rep 2000;49:535-8.

35. Sherer DM. Adverse perinatal outcome of twin pregnancies according to chorionicity: review of the literature. $A m$ J Perinatol 2001;18:23-37. 